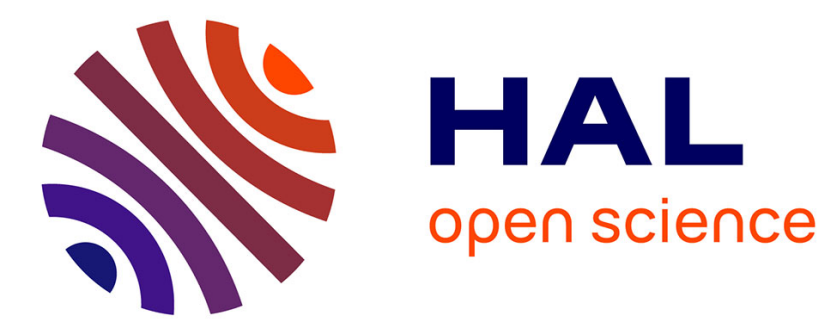

\title{
Les tontes de la Libération en France
}

Fabrice Virgili

\section{To cite this version:}

Fabrice Virgili. Les tontes de la Libération en France. Les cahiers de l'IHTP, 1995. halshs-01939042

\section{HAL Id: halshs-01939042 \\ https://shs.hal.science/halshs-01939042}

Submitted on 29 Nov 2018

HAL is a multi-disciplinary open access archive for the deposit and dissemination of scientific research documents, whether they are published or not. The documents may come from teaching and research institutions in France or abroad, or from public or private research centers.
L'archive ouverte pluridisciplinaire HAL, est destinée au dépôt et à la diffusion de documents scientifiques de niveau recherche, publiés ou non, émanant des établissements d'enseignement et de recherche français ou étrangers, des laboratoires publics ou privés. 
Les Cahiers de l'IHTP, François Rouquet et Danièle Voldman (dir.), Identités féminines et violences politiques (1936-1946), n³1, octobre 1995

Fabrice Virgili, « Les tontes de la Libération en France », p.53-66.

Lors du début du séminaire «Violence, répression et différence des sexes» il y a trois ans, on pouvait légitimement se poser la question : peut-on faire une histoire des tondues ? S'agissait-il de quelques anecdotes largement amplifiées par la mémoire, au point de devenir un mythe ? Une première étude fondée sur la presse[1] de la période laissait déjà supposer la réalité et l'ampleur du phénomène. Sur plus d'une centaine de journaux étudiés pour la période de l'immédiat après-Libération, des tontes étaient mentionnées dans quarante-six départements, et de manière suffisamment nombreuse et variée pour donner une consistance à cette pratique et en faire un véritable objet de recherche[2].

La représentation de ce phénomène par la presse de l'époque apportait un certain nombre de pistes. La tonte, loin d'être une simple «image » de l'épuration, constituait le terrain d'un formidable enjeu de réappropriation et impliquait ainsi des communautés urbaines ou villageoise toutes entières. En offrant la possibilité à chacun de passer de l'événement subi à l'événement vécu, la tonte offre un champ entier à l'étude de la violence et de la différenciation des sexes, ceci dans une étroite imbrication entre vie privée et publique, où la sexualité, le fantasme et l'imaginaire prennent une place non négligeable.

Mais la presse offrait une vision trop étroite pour aborder réellement ces aspects essentiels des tontes. L'étude de fonds d'archives, parfois encore jamais consultés[3], ou de documents pour lesquels cette dimension n'avait pas jusqu'alors été abordée, a permis de prolonger les analyses fondées sur le dépouillement des journaux. Ces sources, extrêmement riches dans les mentions qu'elles offrent, sont malheureusement lacunaires. Du fait de leur conservation tout d'abord, puisqu'un grand nombre d'archives de brigades de gendarmerie ont été détruites dans les années 1950. Elles sont donc inexistantes dans certains départements, rares dans d'autres, nombreuses mais toujours incomplètes ailleurs. L'autre problème tient à la nature même de ces sources. Les dossiers de Cour de Justice, les rapports de gendarmerie, existent quand il y a enquête sur les agissements de ces femmes pendant l'Occupation, ou plus rarement si ces dernières ont porté plainte contre leurs «tondeurs ». Sont ainsi omises toutes celles pour qui l'épuration s'est arrêtée à la coupe de leurs cheveux.

En ce qui concerne les rapports des préfets ou des commissaires régionaux, le problème est d'ordre chronologique. Leurs synthèses, mensuelles ou bi-mensuelles, ne commencent le plus souvent que plusieurs semaines après la Libération, soit après la plus grande vague de tontes. D'autre part ces rapports destinés au gouvernement sont souvent le résultat d'autres synthèses effectuées par les sous-préfets, les services de police ou la gendarmerie. Sur quels critères un événement est sélectionné ou rejeté, quelle est la part des faits qui mérite l'attention du supérieur hiérarchique ? Enfin, et c'est le point commun à tous ces dossiers, ils sont le produit d'une institution, avec leur écriture propre, et leurs préoccupations du moment.

Pourtant l'importance des mentions, la variété de leurs origines, offrent sans conteste une base suffisamment sérieuse pour effectuer cette histoire des tontes à la Libération.

Il n'est nullement question ici d'aborder tous les aspects de cette recherche, mais d'essayer de montrer en quoi les tontes constituent une «évidence » de la Libération. «Évidence» par leur ampleur, par l'importante charge symbolique qu'elles comportent, par le caractère de proximité de cet événement pour de nombreux habitants de ce pays. «Evidence » a posteriori, par la place qu'elles occupent dans la mémoire de cette période et pour les sortir de la marginalité dans laquelle elles semblaient enfermées depuis. Cette pratique acquiert ainsi une certaine légitimité pour ses contemporains. Légitimité donnée à une violence qui frappe essentiellement les femmes tout en conservant une part du caractère politique des affrontements de la période.

Avant d'avancer plus loin dans cet article une réserve de taille : pour l'instant on reste davantage dans le registre du comment que du pourquoi, et les ébauches d'interprétations sont plus des hypothèses que des conclusions. Elles ont pour objectif de faire progresser ce travail tout en confirmant qu'une histoire des tondues est non seulement possible mais nécessaire.

\section{Géographie quantitative et chronologique}


Chiffrer de manière précise et exhaustive les tondues à la Libération est à l'heure actuelle impossible et paraît malheureusement peu envisageable à l'avenir. Aucun recensement des femmes soumises à la tonte n'a été fait. Étant donné le temps et l'énergie nécessaires pour estimer le nombre des victimes de l'épuration, espérer obtenir celui des tondues paraît bien illusoire. Cette lacune ne doit pourtant pas cacher l'ampleur du phénomène. Plusieurs éléments permettent en effet de souligner le caractère massif des tontes.

C'est avant tout l'ensemble du territoire qui est concerné par cette pratique. Soixante-dix-sept départements, selon l'état actuel des dépouillements, ont connu avec certitude des tontes de femmes[4].

L'importance de la couverture géographique implique des régions dont les situations sont fort variées. Il s'agit ainsi autant des premières régions libérées, comme la Normandie et la Corse, que de l'Alsace occupée jusqu'à l'hiver 1945. Elles ont eu lieu dans des territoires libérés aussi bien par les armées alliées, que par la 1ère Armée française, ou encore par la Résistance. Du Limousin et de la Savoie à forte présence de maquis mais aussi de la Picardie. Si une étude plus fine pourra mettre en corrélation ces différents paramètres, il ne semble pas exister un caractère local d'ordre politique, culturel ou historique suffisamment fort pour empêcher le déroulement des tontes à l'échelle d'une région.

Cette omniprésence de tontes est aussi confirmée par leur présence en milieu rural comme dans les zones urbaines. La presse, les rapports de préfets[5] ou des commissaires de la République[6] offrent en général une surreprésentation de l'urbain, ils sont tout à fait complémentaires des archives de la gendarmerie qui au contraire relatent pour l'essentiel les tontes en milieu rural[7].

Dans l'Oise[8], la quantité des mentions permet d'avancer quelques données chiffrées qui confirment l'importance des tontes. On dénombre plus de vingt communes concernées. Il s'agit aussi bien de Beauvais, des villes de second rang comme Noyon, Méru ou Chantilly, que de villages ou de hameaux. On arrive ainsi assez facilement à un ordre de grandeur de cent-cinquante tondues dans ce département.

En Charente-Inférieure les sources trop lacunaires n'offrent pas autant de cas, pourtant La Libération de l'Aunis et de la Saintonge[9] signale que : «Dans un petit village des gamins jouent au maquis... Armés jusqu'aux dents de sabres de bois [...] ils s'emparent du verger, pénètrent au poulailler et libèrent les lapins... Puis ils 'tondent' trois petites filles ». Quand les enfants adoptent pour leurs jeux le comportement de leurs aînés, on a un indice tout aussi significatif de l'extension du phénomène. Indice d'ailleurs confirmé par six autres mentions dans le même journal, et une dizaine de procès-verbaux pour deux brigades de gendarmerie seulement faisant état de tontes[10].

Le deuxième élément qui donne aux tontes ce caractère d'évidence est le nombre parfois considérable de femmes tondues dans un même lieu. À Beauvais[11] la presse locale fait état de quatre-vingt tondues le jour de la Libération, à Lyon, Georgette Elgey[12] rappelle de mémoire les camions entiers qui se dirigent vers la prison de Montluc. Un autre témoin mentionne pour Lille des charettes de tondues et à Chatou on dénombre facilement une trentaine de femmes tondues[13]. Même si l'importance des chiffres nuit probablement à une comptabilité précise, l'ordre de grandeur apporté par certains témoignages est de plusieurs dizaines de femmes. Et l'on peut ajouter à ces exemples, la multiplication de mentions numériquement imprécises « des », « de nombreuses », ou «plusieurs », il existe bien dans de nombreuses villes de France des cortèges de tondues qui constituent un des moment fort de ces journées de la Libération.

Le troisième aspect qui fait de ces coupes de cheveux autre chose qu'un simple épiphénomène, qu'une manifestation spontanée, violente et brève, est justement sa durée. Les tontes durent bien au delà des « journées de la Libération » et l'on peut à la fois parler d'un caractère immédiat et prolongé.

Caractère immédiat, car nombreuses sont celles qui se déroulent dès le moment où la région libérée[14]. Elle paraissent même souvent avoir un caractère urgent de tâche prioritaire. à Chablis[15], «le 25 août, les FFI prennent la ville, installent leur PC à la Mairie et organisent des postes de garde. [...] Ces mesures préventives prises, il fut procédé, sur le seuil de la Mairie et devant une foule en liesse, à la tonsure de huit jeunes personnes qui s'étaient signalées pendant l'occupation par leur sympathie non déguisée à l'égard des boches »;. Et plus loin «le 2 septembre fut procédé à différentes arrestations ", soit huit jours après les tontes. à Trie-Château dans l'Oise[16], «Le 30 août, dès l'arrivée des troupes alliées les résistants se partagèrent en deux groupes. Le premier se mit en campagne pour effectuer la chasse aux boches, le second se mit à la recherche des femmes désignées comme ayant entretenu des relations coupables avec les Allemands ». Dans ces deux cas, la tonte se déroule dans un climat d'incertitude du fait de la présence d'Allemands dans les parages. La recherche et le châtiment des 
« collaboratrices horizontales » sont simultanés à la prise de contrôle du territoire communal, à son «nettoyage » des éventuels soldats ennemis en retraite.

Ce caractère immédiat soulève bien des questions. Comment expliquer la diffusion d'une pratique aussi particulière dans un pays dont tous soulignent l'émiettement? «Pendant l'été de 1944, la France se retrouve atomisée, morcelée en de multiples territoires n'entretenant que peu de contacts entre eux et connaissant tous une chronologie particulière »[17]. Les éléments les plus mobiles de cette période, qu'il s'agisse d'Allemands en retraite, de groupes FFI, des armées françaises ou alliées, ont-ils constitués un vecteur de cette pratique, soit en transmettant des informations sur les régions déjà libérées, soit en l'exécutant eux même ? Quel impact ont pu avoir les récits de tonte diffusés par Radio Londres les 20 et 30 août 1944[18]? Quelle est en effet la place de la spontanéité dans de telles situations, ces actions offrant tous les critères de la préméditation. Si ces tontes sont préparées, ou en tout cas prévues, y-a-t-il place pour un modèle, et quel fut son mode de formation et de propagation?

Si ce sont les tontes de la Libération qui sont le plus souvent restées dans les mémoires, elles se poursuivent pourtant de longs mois encore, la dernière mention étant, pour l'instant, celle d'une tonte en Savoie en février 1946.

Il y a tout d'abord des villes que l'on pourrait qualifier de « retardataires » par rapport à leur libération. à Tournon (Drôme) le journal local des FTP annonce fin septembre au sujet de la tonte de quatre à cinq femmes : «Il n'est jamais trop tard pour bien faire. La ville de Tournon se devait d'avoir aussi ses tondues »[19]. Cela se passe près d'un mois après la Libération, alors que dans les villes voisines de Privas ou deRomans, des tontes ont eu lieu début septembre. Le modèle est ici importé des villes voisines, la tonte semble à Tournon être davantage l'achèvement nécessaire d'une Libération, qu'un acte fondateur de cette libération par la communauté locale. Le phénomène décroît mais sans disparaitre totalement au long de l'hiver 1944-1945. Un certain nombre de femmes ont échappé à la tonte en se cachant lors des journées de la Libération. Une fois revenues dans leur ville, elle ne peuvent pas toujours éviter les enquêtes pour « agissements antinationaux » ou pour « aide directe ou indirecte à l'Allemagne »; elles ont néanmoins ainsi préservé leur chevelure.

Par contre en mai-juin 1945 on peut parler d'une véritable deuxième vague de tontes un peu partout en France . Sur les quais de nombreuses gares, la population attend, dans un sentiment partagé entre la joie des retrouvailles, l'inquiétude de ne pas retrouver à la descente du train la personne tant désirée, la stupeur devant l'état physique et moral des déportés et la découverte de l'horreur du système concentrationnaire nazi ; mais aussi avec la ferme volonté de châtier celles et ceux qui sont partis comme travailleurs volontaires ou se sont enfuis lors de la retraite allemande. Les quais deviennent alors parfois des nouveaux lieux de tonte et de lynchage, les maisons à nouveau occupées depuis le retour de ces collaborateurs sont visées, des prisonniers mis au courant de l'attitude de leur femme, des déportés retrouvent leur dénonciateur. L'épuration légale apparait à nouveau trop lente, laxiste et incomplête. L'épuration extrajudiciaire sous toutes ses formes prend pour quelques semaines une nouvelle ampleur.

Dans seize départements au moins, des tontes parfois nombreuses ont lieu[20]. Le préfet du Jura s'inquiète dans son rapport bimensuel «pour la première fois depuis de nombreux mois et dans différents centres [Lons-leSaunier, Dôle, Champagnole, Salins, Arbois] du département on s'est emparé de certaines femmes pour leur couper les cheveux »[21]. Dans le Jura comme dans les autres départements sont visées des femmes de retour d'Allemagne, qui avait jusque là échappé à l'épuration ; mais aussi des internées administratives depuis plusieurs mois et libérées à ce moment. Cette période passée, ce qui subsiste de l'épuration extra-judiciaire semble se dérouler de manière plus clandestine. Le mitraillage nocturne de maisons, ou l'attentat à l'explosif quand ils visent des femmes, remplaçent d'une certaine manière la coupe des cheveux dans une période où la réprobation semble l'emporter et la poursuite par les autorités de ces actes devenir plus systématique.

La formidable extension de cette pratique peut difficilement être envisagée sans une large approbation des populations, cet aspect renforçant le caractère d'évidence défini plus haut. Il existe pourtant des résistances et des condamnations face au caractère général de cette pratique. Sartre dans sa rubrique «Un promeneur dans Paris insurgé » du journal Combat exprime son «dégoût » face à ce "sadisme moyenâgeux »[22]. Le journal communiste des Bouches-du-Rhône La Marseillaise dénonce « des procédés qui rappellent précisément les pires habitudes des ignobles sadiques SA et SS «, même si une semaine auparavant il vantait les « vaillants FFI » qui avaient arrêté des femmes vues en compagnie d'Allemands pour leur raser la tête[23]. Dans la Dépêche de l'Aisne ou L'écho de la Corrèze, des articles en appellent à l'égalité entre hommes et femmes devant l'épuration. Presque partout on constate des appels de la part des FFI, FTP, CDL, ou des autorités gouvernementales pour 
cesser toute brimade. Pourtant ces discours hostiles aux tontes, et qui tentent de les exclure de la Libération au nom d'une certaine morale, ne paraissent pas ôter leur légitimité à celles-ci.

\section{Légitimation des tontes}

On ne peut parler d'une légitimité incontestablement reconnue. De la part des autorités, il n'y a pas de trace d'une loi, d'un appel national - qu'il émane de De Gaulle, du gouvernement, ou de la Résistance - pour tondre les femmes accusées de collaboration. De la part de la presse, si les articles en faveur des tontes sont légèrement majoritaires, il n'y a pas d'appel à la coupe des cheveux des collaboratrices. Cette légitimité semble bien plus se constituer simultanement aux tontes, être le résultat d'un entrecroisement de discours et de pratiques qui constituent un système de représentation dans lequel la coupe au rasoir des cheveux apparaît comme un châtiment proportionné, juste, efficace et nécessaire.

Le premier type d'arguments permet de rappeler le contexte dans lequel se déroulent ces tontes. Celles-ci sont mise en perspective avec les horreurs du nazisme. « Notre conscience de civilisé se fut autrefois révoltée contre cette formule de répression spectaculaire digne d'un autre temps. Notre sensibilité aurait-elle été donc à ce point émoussée par la barbarie teutonne? Je ne le pense pas. Mais notre indignation fut telle au cours de ces quatre années qu'aujourd'hui notre colère explose »[24]. Ce point de vue de L'écho de la Corrèze illustre bien le désarroi de cette situation de crise prolongée dans laquelle les repères ont disparu et les cadres totalement bouleversés. Tout en analysant cette pratique comme anachronique - on retrouve cette accusation dans le « moyenâgeux » employé par Jean-Paul Sartre dans sa rubrique - l'auteur en légitime les formes par l'atmosphère générale du temps. Vécu de souffrance, non seulement extrême comme celle qui auréole à juste titre ceux qui sont morts en connaissant le pire, mais aussi vécu de toutes les frustrations, de la faim et de l'angoisse, ou de l'humiliation. Souffrance du plus grand nombre à laquelle ont échappé, ou en tout cas semblent avoir échappé pour la communauté, ces femmes pour qui c'est désormais le tour. « Journée de liesse à Châteauroux, journée où les femmes ont eu leur revanche. Les beaux cheveux de quelques unes sont tombés. C'est tant mieux, à chacun son tour de trembler » peut déclarer l'Union des femmes françaises de l'Indre ; et d'ajouter, « les agissements des misérables et infâmes créatures déshonorées et corrompues par l'ennemi était un défi au vu du sacrifice supporté par les femmes françaises $»[25]$.

On retrouve là toutes les contradictions de la représentation des femmes à la Libération et la difficulté de cerner cette image entre deux modèles totalement opposés. Affirmer que les femmes ont eu leur revanche n'est pas sans soulever toute une série de questionnement sur la finalité des tontes. Les femmes, au-delà de celles qui sont tondues, ne sont-elles pas aussi indirectement victimes de ces actions? Les descriptions, commentaires et témoignages empruntent trop souvent à un répertoire mysogine pour que ces propos concernent les seules collaboratrices. La dénonciation de l'insouciance, de la légèreté et du goût du luxe, la stigmatisation surtout d'un comportement de mauvaise fille, de mauvaise épouse ou de mauvaise mère semblent s'adresser à toutes les femmes. L'acquisition de la citoyenneté s'accompagnerait-elle ainsi d'un rappel du rôle traditionnel dans lequel les femmes doivent être maintenues ? Cela n'allait pas sans le risque d'entâcher la participation et le combat des Françaises pour la Libération du pays. D'où la nécessité pour les Français de l'été 1944, d'exclure les « tondues » de la communauté locale et nationale, de les nier également en tant que femmes, préservant ainsi en partie mais en partie seulement cette image de la Française vertueuse et courageuse.

Tondre c'est avant tout punir, épurer. Au contexte général de la guerre et de l'occupation, s'ajoute celui de la Libération et de ces urgences, «Vaincre, punir, reconstruire »[26]. La tonte permet à chacun de participer à cette urgence, elle est un événement de proximité vécu par tous ou presque. Contrairement aux procès, plus tardifs et clos dans l'univers des salles d'audience, à l'internement lui aussi inscrit dans la durée et l'enfermement, les tontes et certaines exécutions, permettent à la communauté urbaine ou villageoise d'être le témoin immédiat et direct, et donc le garant, de cette politique d'épuration, de s'en approprier ainsi l'exécution.

Les formes de cette violence sont pourtant multiples, car il y a autant de manière de tondre que d'épurer.

Il existe d'abord, et ce sont les premières dans le temps, des « tontes au coin du bois ». Nous n'en avons pas parlé pour l'instant, mais les tontes commencent avant la Libération, elles se déroulent alors de manière clandestine, le plus souvent de nuit à l'intérieur du domicile de collaboratrices dont les biens sont en même temps récupérés. Sur les auteurs rien, si ce n'est parfois une mention signalant que le motif «pourrait être d'ordre politique ». En Loire-Atlantique début mars 1944, en Ille-et-Vilaine en mai, ou en Isère en juin, ces tonte se ressemblent. Par contre à Lyon la coupe des cheveux d'une femme "réputée collaboratrice » s'est accompagnée de son exécution[27]. Mais, si comme le souligne Henry Rousso, « $80 \%$ des exécutions dites ' sommaires ' ont été 
perpétrées pour partie en pleine Occupation, pour l'essentiel au moment des combats de la Libération », les tontes se déroulent le plus souvent, comme nous l'avons vu, une fois la Libération effective[28]. Ce décalage renforce l'interprétation des tontes comme événement à forte valeur symbolique et collective, qui dépasse le simple règlement de compte. Pour que la tonte joue son rôle de châtiment expiatoire et de ciment communautaire, elle doit être ostentatoire. Condition pratiquement impossible à remplir avant la Libération.

à l'opposé de ces tontes clandestines, se pratiquent aussi des tontes quasi-officielles. à l'intérieur des lieux de pouvoir ou de coercition (préfectures, mairies, commissariats, prisons), la tondeuse fonctionne aussi. C'est le cas à Romans (Drôme) où le 6 septembre 1944 dix détenues ont les cheveux coupés[29], dans la prison Saint-Joseph à Grenoble, où «le coiffeur de la prison [avait] fait fonctionner sa tondeuse selon le goût du jour pour ces dames»[30]. Caractère «officiel» qui voit le tribunal d'exception siégeant au Palais de Justice de Tulle condamner une jeune fille à « 3 mois de prison avec coupe totale des cheveux », alors que le commissaire du gouvernement avait requis 20 ans de travaux forcés[31].

C'est pourtant la tonte publique, appropriée collectivement, qui reste la plus fréquente, et constitue également pour la mémoire une des images de la Libération. Sans pouvoir pour l'instant parler d'un modèle reproduit à travers tout le territoire, il faut noter que ces tontes ont de nombreux points communs. Avec les défilés des armées alliées, celui des FFI, les cérémonies au monument aux morts, les bals, l'arrestation ou le transfert des collaborateurs, les tontes s'intègrent dans un système d'affirmation de la collectivité et de construction d'une nouvelle identité commune.

L'espace public est dans cette perspective le lieu privilégié de la tonte. à Moulins, une femme est directement tondue sur son balcon. Le balcon lieu privilégié de la séduction, depuis qu'une célèbre Juliette y attendit son prétendant, se transforme ici en scène ou plutôt en échafaud afin que la tonte se déroule à la vue de tous. Les lieux peuvent varier mais ils sont en général référents pour la population. Que ce soit devant la mairie, sur la place du marché ou à la fontaine du village, la promenade doit permettre de se réapproprier cet espace collectif confisqué pendant quatre années par la peur, le couvre-feu, les affiches, drapeaux, inscriptions, et bien sûr par la police vichyste, la Milice et les troupes d'occupation.

La similitude des rites, la mise en place d'un «bureau de tonte », qu'il se trouve dans un café, sur le quai d'une gare, ou sur une estrade, l'appel au coiffeur de la localité et à ses outils, sont autant de signes d'une organisation de cette violence.

$\mathrm{Ce}$ «spectacle» auquel participe la population peut avoir de nombreuses références[32]. Une perspective anthropologique permet d'y déceler les ressemblances avec les carnavals[33], le châtiment des femmes adultères au Moyen-âge[34], ou encore les exécutions d'ancien régime[35]. à Tulle, l'auteur d'un billet intitulé «Un pittoresque défilé » sur la promenade d'une tondue, fait directement référence aux défilés des « Jeudis gras » de son enfance sur cette terre d'élevage du Limousin[36]. On peut aussi voir dans « la tondue » l'effigie brûlée ou pendue de certaines manifestations. La coupe des cheveux étant alors une manière de donner une mort symbolique à la collaboration.

\section{Châtiment de la collaboration ou punition des femmes ?}

La tonte occupe une place particulière dans le châtiment de la collaboration, mais n'est pas exclusive d'autres condamnations. Si rares sont les femmes tondues et exécutées, bien plus nombreuses sont celles pour qui l'internement administratif, la condamnation, à l'emprisonnement en Cour de Justice, à l'indignité nationale ou l'interdiction de séjour en Chambre civique s'ajoute à leur crâne rasé. Si des femmes sont libérées une fois leurs cheveux coupés, d'autres n'en sont pas quittes avec l'épuration. Dans les dossiers des commissions d'épuration comme dans ceux des tribunaux, on retrouve régulièrement une mention de tonte lors de la Libération. Certaines sont tondues, libérées, puis à nouveau arrêtées. La coupe des cheveux devenant parfois une preuve a posteriori de la conduite reprochée à ces femmes, elle devient ainsi, comme la marque au fer rouge de l'Ancien Régime, le signe de la culpabilité, et désigne à la vue de tous la collaboratrice. C'est le cas dans ce témoignage à charge extrait du dossier d'une femme poursuivie pour inconduite notoire : «J'ai appris dernièrement que les FFI de Marseille en Beauvaisis lui avaient coupés les cheveux, preuve sans aucun doute de sa bonne conduite »[37].

La tonte apparait comme une violence spécifique visant les femmes, sans effet sur d'éventuelles autres poursuites. Cela indépendamment de la nature du crime reproché : dénonciation d'un résistant, travail pour les Allemands, participation à une organisation de la collaboration, relation sexuelle avec les Allemands. 
«Y a-t-il une loi française qui condamne à la tondaison, et les femmes seules? » interroge une journaliste de l'écho de la Corrèze[38]. Non, pourtant cette pratique et le discours qui l'accompagne visent essentiellement les femmes et ne peuvent avoir ainsi pour seule fonction de châtier un acte de collaboration.

La mise en scène des tontes, les discours qui les commentent, la description des femmes accusées de collaboration imprègnent le politique de fantasme, de sexualité et d'imaginaire. Sont stigmatisés la prostitution de celles qui ont vendu leur corps aux Allemands comme l'adultère de celles qui ont trahi leur mari quand celuici était prisonnier. Mais dans une sorte de glissement où l'image de la femme s'apparente à celle de Marianne et donc à la Nation, ces femmes sont finalement accusées d'avoir permis la souillure du pays par celle de leur propre corps. De manière extrêmement violente, c'est ce qu'affirme le Procureur général de Besançon quand il prend en considération « la possibilité même d'un mélange effectif du sang français et de la race allemande, dont certaines affaires ont malheureusement montré la pénible réalité, mélange qui porte une atteinte réelle que l'on pourrait qualifier de matérielle, à l'unité de la nation française »[39].

Avec les descriptions des collaboratrices horizontales, qui n'hésitent pas à emprunter à un répertoire pornographique, dans les scènes de tonte où l'érotisation est omniprésente, on assiste à un processus de destruction d'une double image, celle de Françaises et celles de femmes. De Françaises, car il s'agit bien de les exclure de la collectivité nationale, en les opposant aux femmes résistantes, aux femmes françaises en général désormais citoyennes. Mais aussi de femmes, puisque c'est leur corps qui est châtié, corps devenu objet du délit la collaboration -, porteur du châtiment - la tonte - mais corps qui doit aussi, dans la perspective de la reconstruction et du redressement, se soumettre. Le corps de ces femmes ne leur appartient plus, il devient celui de la Nation. Dans une période où se manifeste une culture combattante, guerrière et virile, le corps de la « tondue » devient un enjeu de pouvoir. «La France sera virile ou morte » peut ainsi conclure un article intitulé « La loi virile »[40].

Et pourtant les femmes ne sont pas les seules victimes de ces tontes, des hommes aussi sont tondus, de manière certaine dans au moins sept départements[41]. Cela n'enlève pourtant rien au caractère défini ci-dessus. Ces cas sont tout d'abord très peu nombreux. Le caractère sexuel n'est jamais mis en avant dans les faits reprochés ; ces hommes sont tondus selon les cas pour pillage, travail en Allemagne, appartenance à des groupes de la collaboration (PPF, Milice, Franciste). La sanction s'appliquant à eux en même temps qu'aux femmes présentes, on peut l'interpréter comme un moyen supplémentaire de les humilier en les assimilant à ces dernières. Ainsi dans le Tarn-et-Garonne, quelques hommes et gradés n'ayant pas accepté de partir combattre sur le front, « leurs camarades ont exercé quelques brimades. Ils ont tondu les cheveux des " défaillants " après les avoir promenés dans les rues de la ville $»[42]$.

N'est-il pas paradoxal de conclure un article sur les «tondues » par le cas des hommes tondus ? Je ne le pense pas, les tontes de la Libération ont ceci de particulier, qu'elles ne peuvent être enfermées dans un domaine spécifique de l'histoire. Il ne s'agit pas de l'histoire des femmes, d'une lecture «sexuelle » de la période, mais bien d'un objet historique riche de sens pour la compréhension de cette époque. Au croisement de domaines aussi variés que l'étude de l'opinion, l'histoire culturelle, les questions juridiques, l'histoire politique, l'anthropologie, l'histoire quantitative, ou orale, les «tondues» obligent à d'incessants allers-retours entre différentes grilles de lecture.

C'est ainsi que l'on parviendra peut-être à comprendre cet événement exceptionnel. Exceptionnel, par les effets qu'il provoque, mais aussi par son " originalité », son aspect presque anachronique. Comment ne pas être surpris de voir, au milieu de notre siècle, la population d'un pays se consacrer à couper les cheveux de milliers de femmes?

Notes :

[1] Je renvois ici à mon DEA «Tontes et tondues à travers la presse de la Libération », Université de Paris 1Sorbonne, 1992, sous la direction d'Antoine Prost et de Jean-Louis Robert. Consultable à l'IHTP ou au Centre de recherches d'histoire des mouvements sociaux et du syndicalisme.

[2] Cent-onze journaux couvrant soixante-treize départements pour le mois qui suivait la Libération de chacun d'entre eux.

[3] Il s'agit des archives des Cours de justice et Chambres civiques de l'Oise. L'autre source essentielle est celle de la gendarmerie nationale dont les archives se trouvent au Blanc (Indre). 
[4] Sur quatre-vingt-dix départements à l'époque. Manquent probablement plus par le fait des hasards de la recherche que par absence véritable de l'événement : l'Ain, les Hautes-Alpes, le Calvados, le Cher, l'Eure, le Gers, la Haute-Loire, la Haute-Marne, la Mayenne, la Meuse, l'Orne, le Haut-Rhin, la Vendée, le Territoire de Belfort. Cette liste n'est bien-sûr pas définitive et toute information permettant de la réduire est évidemment bienvenue.

[]] AN F1cIII/1205 à 1233.

[] AN F1a/4021à4028.

[7] Centre administratif de la gendarmerie nationale - Le Blanc (Indre).

[] L'Oise est pour l'instant le seul département pour lequel une recherche exhaustive est en cours. L'étude porte sur la presse, les rapports du prefet, les procès-verbaux de Gendarmerie, les archives des commissions d'épuration, des Chambres civiques et de la Cour de justice.

[9] La Libération de l'Aunis et de la Saintonge, 14 octobre 1944.

[10] Il s'agit des brigades de Rochefort et de Montlieu-la-Garde.

[11] L'Oise Libérée, 2 septembre 1944.

[12] Georgette Elgey, « La fenêtre ouverte », Paris, 1947.

[13] Sources privées.

[14] Deux tiers des 300 mentions de tontes concernent ces « journées ».

[15] Yonne Républicaine, 27 septembre 1944, information recoupée avec un PV de Gendarmerie de la brigade de Chablis du 6 décembre 1944.

[16] AD Oise, 34W845.

[17] Philippe Buton «La France atomisée », dans Jean-Pierre Azéma, François Bédarida, (dir.), La France des Années noires, Paris, Seuil, 1993.

[18] Jean-Louis Crémieux-Brilhac « Ici Londres\&nbsp:», Paris, Documentation française, 1976, vol. 5, pp. 191 et 226.

[19] L'Assaut, 25 septembre 1944.

[20] Allier, Aveyron, Charente, Corrèze, Indre, Indre-et-Loir, Jura, Loiret, Lot, Maine-et-Loire, Morbihan, Moselle, Oise, Puy-de-Dôme, Haut-Rhin, Vosges.

[21] AN F1cIII/1219.

[22] Combat, 2 septembre 1944.

[23] La Marseillaise, 1er septembre 1944.

[24] L'Echo de la Corrèze, 14 septembre 1944.

[25] AD Indre, Le Département, organe du CDL, 25 Août 1944, Châteauroux.

[26] Philippe Buton, «L'État restauré », dans La France des années noires, op. cit.

[27] AN BB18 / 3618 Le corps est découvert en octobre, l'exécution semble remonter dans ce cas aux jours précédant la libération de la ville.

[28] Henry Rousso, «L'épuration en France une histoire inachevée », Vingtième siècle. Revue d'histoire, $\mathrm{n}^{\circ} 33$, janvier-mars 1992, pp. 78-105.

[29] Le Patriote Romanais, 8 septembre 1944.

[30] Les Allobroges, 5 septembre 1944.

[31] L'Echo de la Corrèze, 10-11 septembre 1944.

[32] Ce terme de spectacle est largement utilisé dans la presse de l'époque

[33] Alain Brossat, Les tondues. Un carnaval moche, Paris, Manya, 1992, et plus particulièrement le chapitre «Un bruit surgi du fond des âges », pp. 247-256.

[34] Jean-Marie Carbasse, « 'Currant nudi', la repression de l'adultère dans le Midi médiéval (XII- XV) 》 dans J. Poumarède et J.-P. Royer (dir.), Droit, Histoire et Sexualité, Paris, L'espace juridique, 1987, pp. 83-102.

[35] Michel Foucault, Surveiller et punir, Paris, Gallimard, 1975, p. 47.

[36] L'Echo de la Corrèze, 14 septembre 1944.

[37] AD Oise 34W8466, dossiers du cabinet du préfet.

[38] L'Echo de la Corrèze, 18 septembre 1944. Le nombre d'extraits tirés de ce journal peut surprendre, il s'explique par la publication au long du mois de septembre de trois articles contradictoires sur la même tonte. Chose suffisemment rare pour être notée.

[39] AN BB18/7133, correspondance du 28 février 1945 du Procureur général à la Direction criminelle de la cour d'appel de Besançon. Des propos de ce type sur la pureté du sang et la souillure que constitue la naissance d'un enfant de père allemand restent rares à la Libération, il furent par contre, comme le montre Judith Wishnia, l'objet d'un véritable débat public pendant la guerre de 14-18. Judith Wishnia, "Natalisme et nationalisme (1914-1918) », Vingtième siècle. Revue d'histoire, n 45 janvier-mars 1995, pp. 30-39.

[40] Renaissance républicaine du Gard, 30 août 1944.

[41] Allier, Aveyron, Drôme, Isère, Loire, Loiret et Tarn-et-Garonne.

[42] AN F1a / 4028 Rapport du 31 décembre 1944 du commissaire régional de la République. 
